\title{
The relationship between depressive symptoms and dietary intake in people at high risk of cardiovascular diseases
}

\author{
W. Alfawaz ${ }^{1}$, N. Guess ${ }^{2}$, K. Ismail ${ }^{1}$ and K. Stewart ${ }^{1}$ \\ ${ }^{1}$ Institute of Psychiatry, Psychology \& Neuroscience, Department of Psycological medicine, King's College London, \\ SE5 $9 R J$ and \\ ${ }^{2}$ Divisions of Diabetes and Nutritional Sciences, King's College London, SE1 9NH
}

There is a bilateral association between depression and diet ${ }^{(1)}$. There is some evidence suggests that unhealthy diets are associated with an increased risk developing depression ${ }^{(2)}$ but there is less evidence that depression is associated with worsening of diet. Understanding the role of depression on dietary behaviours may help reduce the risk of obesity, type 2 diabetes and cardiovascular disease (CVD) ${ }^{(3)}$. The overall aim of this study is to test whether depression is prospectively associated with unhealthy diets, and whether there is a pattern in the type of nutrient, the amount and timing of ingestion.

This study consists of a series of secondary analyses set in the MOVE-IT cohort (the enhanced MOtiVational intErviewing InTervention) which tested the effectiveness of an intensive healthy lifestyle intervention for people at high risk of CVD over a twoyear period. The MOVE-IT was a three-arm, parallel RCT comparing the effectiveness of a one-year lifestyle intervention (delivered in group and individual formats) versus usual care in reducing weight and increasing physical activity. Participants were adults aged between 40 and 74 years recruited from primary care settings in South London.

Depressive symptoms was evaluated using PHQ-9 and 24-hour recall method was used to assess dietary intake. Dietplan7 was used for coding and deriving nutrient intake. Age, gender, ethnicity, BMI, baseline value of nutrient of interest, IMD-10 score, trial arm, and number of intervention sessions were used as covariates. Data was analysed using SPSS 22.

At baseline in the MOVE IT cohort, there was a weak positive association between depressive symptoms and amount of CHO $(p=0.006)$ and total sugar $(p=0.034)$ and negative association between depressive symptoms and alcohol intake $(p=0.012)$. There was a negative association between depressive symptoms and \% Kcals from CHO $(\mathrm{p}=0.016)$ and positive association between depressive symptoms and alcohol $(\mathrm{p}=0.008)$ at year one. After the second year, this association with $\% \mathrm{Kcal}$ from $\mathrm{CHO}$ disappeared, and we found a positive association instead between depressive symptoms and saturated fat $(\mathrm{p}=0.033)$ and alcohol intake $(\mathrm{p}=0.035)$. No association was found between depressive symptoms and the type of food intake as well as timing of ingestion at each timepoints. The experimental interventions had a null effect on its primary outcomes (weight and PA), it can be concluded with some confidence that in effect the MOVE IT cohort was more akin to a prospective cohort than originally thought.

Our results suggest that depressive symptoms may alter dietary intake but not in a consistent pattern or at a clinically significant level. Future prospective cohort studies in primary care should focus on larger samples of depressed patients.

1. Jacka FN, Cherbuin N, Anstey KJ, Butterworth PJ (2015) Does reverse causality explain the relationship between diet and depression? JoAD 175, $248-50$.

2. Opie R, Itsiopoulos C, Parletta N, Sanchez-Villegas A, Akbaraly TN, Ruusunen A, et al. (2017) Dietary recommendations for the prevention of depression 20(3), 161-71.

3. Kinder LS, Carnethon MR, Palaniappan LP, King AC, Fortmann SP (2004) Depression and the metabolic syndrome in young adults: findings from the Third National Health and Nutrition Examination Survey. JPM 66(3), 316-22. 